$$
\gamma_{h i j}=\gamma_{j i h}
$$

where $i$ takes on a definite set of $n-m$ values of the integers $1,2, \ldots, n$ including the value $k$, and $h$ and $j$ take on those $m$ values that $i$ cannot assume. If each of $n-m$ congruences forms a system of parallels with respect to every one of the remaining $m$ congruences equations (11) are satisfied for $h=1,2,3, \cdots, n ; i=i_{1}, i_{2}, \cdots, i_{n-m}$; $j=j_{1}, \cdots, j_{m} ; i \neq j,(12)$ is surely satisfied, and we have the following theorem.

Theorem. If each of $n-m$ congruences forms a system of parallels with respect to every one of the remaining $m$ congruences, then the former have a family of m-dimensional hypersurfaces as orthogonal trajectories. When $m=n-1$ this reduces to one of Lipka's theorems.

Princeton University

\title{
SUR LES VALEURS ASYMPTOTIQUES DES COEFFICIENTS DE COTES
}

PAR J. OUSPENSKY

1. Parmi les formules de quadratures pour le calcul approché des intégrales définies la plus simple est, sans contredit, celle de Cotes, qui correspond à la division de l'intervalle d'intégration en parties égales. Supposons l'intervalle d'intégration $(0,1)$ subdivisé en $n$ parties égales; alors on peut déterminer $n+1$ constantes $A_{0}, A_{1}, A_{2}, \cdots, A_{n}$, nommées "coefficients de Cotes", de manière que la formule

$$
\int_{0}^{1} f(x) d x=A_{0} f(0)+A_{1} f\left(\frac{1}{n}\right)+A_{2} f\left(\frac{2}{n}\right)+\cdots+A_{n} f(1)
$$

soit exacte pour toute fonction $f(x)$ se réduisant à un polynôme d'un degré n'excédant pas $n-1$. Dans d'autres cas cette "formule de Cotes" n'est qu'approchée. Comme le degré d'approximation fourni par elle depend des valeurs numériques des coefficients $A_{0}, A_{1}, A_{2}, \cdots, A_{n}$, la 
question sur les valeurs asymptotiques de ces coefficients pour $n$ très grand n'est pas, ce me semble, dénuée de quelque interêt.

2. En posant

$$
x_{0}=0, \quad x_{1}=\frac{1}{n}, \quad x_{2}=\frac{2}{n}, \quad \cdots, \quad x_{n}=1,
$$

et

$$
\varphi(x)=x\left(x-\frac{1}{n}\right)\left(x-\frac{2}{n}\right) \ldots\left(x-\frac{n}{n}\right),
$$

on a l'expression suivante pour le coefficient $A_{k}, k$ étant un des nombres $0,1,2, \cdots, n$ :

$$
A_{k}=\int_{0}^{1} \frac{\varphi(x) d x}{\left(x-x_{k}\right) \varphi^{\prime}\left(x_{k}\right)} .
$$

Mais comme l'on sait que

$$
\begin{aligned}
\varphi^{\prime}\left(x_{k}\right) & =\frac{k}{n} \cdot \frac{k-1}{n} \cdots \frac{1}{n} \cdot \frac{-1}{n} \cdot \frac{-2}{n} \ldots \frac{-(n-k)}{n} \\
& =(-1)^{n-k} \frac{\Gamma(k+1)}{n^{n}}, \frac{\Gamma(n-1)}{}
\end{aligned}
$$

on trouve après quelques réductions faciles:

$$
A_{k}=\frac{(-1)^{n-k}}{n \Gamma(k+1) \Gamma(n-k+1)} \int_{0}^{n} \frac{x(x-1) \cdots(x-n)}{x-k} d x .
$$

Ainsi la question se réduit à trouver l'expression asymptotique pour $n$ très grand de l'intégrale:

$$
J_{n}=\int_{0}^{n} \frac{x(x-1) \cdots(x-n)}{x-k} d x
$$

$k$ étant un nombre quelconque de la suite $0,1,2, \cdots, n$. En faisant usage des formules bien connues

$$
\begin{gathered}
x(x-1) \cdots(x-n)=(-1)^{n} \frac{\Gamma(x+1) \Gamma(n+1-x) \sin \pi x}{\pi}, \\
\frac{\Gamma(x+1) \Gamma(n+1-x)}{\Gamma(n+2)}=\int_{0}^{1} \xi^{x}(1-\xi)^{n-x} d \xi,
\end{gathered}
$$

nous allons présenter d'abord $J_{n}$ sous la forme d'une intégrale double: 
$J_{n}=\frac{(-1)^{n} \boldsymbol{T}(n+2)}{\pi} \int_{0}^{1}(1-\xi)^{n} d \xi \int_{0}^{n} e^{x \log \frac{\xi}{1-\xi} \frac{\sin \pi x}{x-k}} d x$.

Ensuite, en utilisant la formule évidente

$$
\begin{aligned}
& \int_{0}^{n} e^{x \log \frac{\xi}{1-\xi} \frac{\sin \pi x}{x-k}} d x=(-1)^{k}\left(\frac{\xi}{1-\xi}\right)^{k} \\
& \times\left[\int_{0}^{n-k} e^{x \log \frac{\xi}{1-\xi} \frac{\sin \pi x}{x}} d x+\int_{0}^{k} e^{\left.x \log \frac{\xi}{1-\xi} \frac{\sin \pi x}{x} d x\right],}\right.
\end{aligned}
$$

et en transformant les intégrales du second membre à l'aide, de la formule

$$
\int_{0}^{\rho h} e^{\alpha x} \frac{\sin \pi x}{x} d x=\frac{\pi}{2}+\arctan \frac{\alpha}{\pi}+\pi(-1)^{h-1} \int_{-\infty}^{\alpha} \frac{e^{h x} d x}{\pi^{2}+x^{2}},
$$

que l'on établit aisément en supposant $h$ un entier quelconque, on parvient à cette expression définitive de $J_{n}$ :

$$
\begin{aligned}
& J_{n}=(-1)^{n-k} \boldsymbol{\Gamma}(k+1) \boldsymbol{\Gamma}(n-k+1)+\boldsymbol{\Gamma}(n+2) \\
& \times\left[(-1)^{n-1} \int_{0}^{1} \xi^{k}(1-\xi)^{n-k} d \xi \int_{-\infty}^{\circ \log \frac{1-\xi}{\xi} \frac{e^{k x} d x}{\pi^{2}+x^{2}}}\right. \\
&\left.\quad-\int_{0}^{1} \xi^{k}(1-\xi)^{n-k} d \xi \int_{-\infty}^{\log \frac{1-\xi}{\xi}} \frac{e^{(n-k) x} d x}{\pi^{2}+x^{2}}\right] .
\end{aligned}
$$

Nous allons faire usage de cette formule en supposant d'abord $k$ différent de 0 et $n$. On voit de suite qu'en posant

$$
\begin{aligned}
& P_{1}=\int_{0}^{\frac{1}{2}} \xi k(1-\xi)^{n-k} d \xi \int_{-\infty}^{\log \frac{1-\xi}{\xi}} \frac{e^{k x} d x}{\pi^{2}+x^{2}} ; \\
& P_{2}=\int_{0}^{\frac{1}{2}} \xi^{k}(1-\xi)^{n-k} d \xi \int_{-\infty}^{\log \frac{1-\xi}{\xi}} \frac{e^{(n-k) x}}{\pi^{2}+x^{2}} d x \\
& P_{3}=\int_{0}^{\frac{1}{2}} \xi^{k}(1-\xi)^{n-k} d \xi \int_{-\infty}^{\log \frac{\xi}{1-\xi}} \frac{e^{k x} d x}{\pi^{2}+x^{2}} \\
& P_{4}=\int_{0}^{\frac{1}{2}} \xi^{k}(1-\xi)^{n-k} d \xi \int_{-\infty}^{\log \frac{\xi}{1-\xi}} \frac{e^{(n-k) x} d x}{\pi^{2}+x^{2}}
\end{aligned}
$$


on a

(3)

$$
\begin{aligned}
J_{n}=(-1)^{n-k} \boldsymbol{\Gamma}(k+1) \boldsymbol{\Gamma}(n-k+1)+\boldsymbol{\Gamma}(n+2) \\
\times\left[(-1)^{n-1} P_{1}-P_{2}+(-1)^{n-1} P_{3}-P_{4}\right] .
\end{aligned}
$$

Mais comme

$$
\begin{aligned}
& \int_{-\infty}^{\log \frac{\xi}{1-\xi}} \frac{e^{k x} d x}{\pi^{2}+x^{2}}<\int_{-\infty}^{\log \frac{\xi}{1-\xi}} e^{k x} d x=\frac{\xi^{k}}{k(1-\xi)^{k}}, \\
& \int_{-\infty}^{\log \frac{\xi}{1-\xi}} \frac{e^{(n-k) x} d x}{\pi^{2}+x^{2}}<\frac{\xi^{n-k}}{(n-k)(1-\xi)^{n-k}},
\end{aligned}
$$

on voit immédiatement que

$$
\begin{gathered}
0<P_{3}<\frac{1}{k} \int_{0}^{\frac{1}{2}} \xi^{n} d \xi=\frac{1}{k(n+1) 2^{n+1}} ; \\
0<P_{4}<\frac{1}{n-k} \int_{0}^{\frac{1}{2}} \xi^{n} d \xi=\frac{1}{(n-k)(n+1) 2^{n+1}},
\end{gathered}
$$

ce qui permet de présenter la formule (3) de cette manière:

$$
\begin{aligned}
J_{n} & =(-1)^{n-k} \boldsymbol{\Gamma}(k+1) \Gamma(n+1-k)+\boldsymbol{\Gamma}(n+2) \\
& \times\left[(-1)^{n-1} P_{1}-P_{2}+\frac{\Theta}{k(n-k) 2^{n+1}}\right],-1<\Theta<1 .
\end{aligned}
$$

Toute la question se réduit ainsi à trouver les expressions asymptotiques de $P_{1}$ et $P_{2}$, ce qui présente un peu plus de difficulté.

3. La solution de cette question dépend en grande partie de la recherche de la valeur asymptotique de l'intégrale

$$
K_{n}=\int_{0}^{c h} \frac{(1-x)^{n} d x}{\left(\log \frac{1-x}{x}\right)^{s}}
$$

pour $n$ indéfiniment croissant, tandis que $h$ est un nombre fixe, satisfaisant aux inégalités

$$
0<h<\frac{1}{2},
$$

et $s$ un nombre entier positif donné. Considérons l'intégrale 
un peu plus générale

$$
K_{n}(\alpha)=\int_{0}^{h} \frac{\left(\frac{1-x}{x}\right)^{\alpha}(1-x)^{n}}{\left(\log \frac{1-x}{x}\right)^{s}} d x
$$

qui dépend d'un paramètre $\alpha$ et se réduit à $K_{n}$ pour $\alpha=0$. Nous allons supposer dans la suite que

$$
-\frac{1}{2} \leqq \alpha \leqq 0
$$

L'inégalité presque évidente

$$
0<\int_{0}^{\rho n} \frac{(1-x)^{n-\frac{1}{2}} x^{\frac{1}{2}} d x}{\left(\log \frac{1-x}{x}\right)^{k}}<\frac{1}{\left(\log \frac{1-h}{h}\right)^{k}} \frac{\Gamma\left(n+\frac{1}{2}\right) \Gamma\left(\frac{3}{2}\right)}{\Gamma(n+2)},
$$

et la formule asymptotique bien connue

$$
\lim \frac{\Gamma(n+\beta)}{n^{\beta} \Gamma(n)}=1, \text { pour } n=\infty,
$$

font voir que pour chaque valeur fixe de $k$, l'intégrale

$$
\int_{0}^{h} \frac{(1-x)^{n-\frac{1}{2}} x^{\frac{1}{2}} d x}{\left(\log \frac{1-x}{x}\right)^{k}}
$$

est de l'ordre $1 / n^{3 / 2}$, c'est à dire qu'il existe une constante $L$ telle que l'intégrale précédente soit $<L / n^{3 / 2}$.

Il suit immédiatement de cette remarque que les valeurs que prennent $K_{n}(\alpha)$ et ses dérivées pour $\alpha=-\frac{1}{2}$ se réduisent aux quantités d'ordre $O(1 / n)^{3 / 2}$. Ensuite la dérivée d'ordre $s$ de $K_{n}(\alpha)$ se present sous la forme

$$
\begin{aligned}
\frac{d^{s} K_{n}(\alpha)}{d \alpha^{s}} & =\int_{0}^{h}(1-x)^{n+\alpha} x^{-\alpha} d x \\
= & \frac{\Gamma(n+\alpha+1) \Gamma(1-\alpha)}{\Gamma(n+2)}-\int_{h}^{1}(1-x)^{n+\alpha} x^{-\alpha} d x .
\end{aligned}
$$

L'intégrale qui figure dans le second membre de cette 
formule est positive et plus petite que $(1--h)^{n-1 / 2}$; de l'autre côté les formules asymptotiques bien connues font voir que la quantité

$$
\frac{\Gamma(n+\alpha+1) \Gamma(1-\alpha)}{\Gamma(n+2)}
$$

peut être présentée ainsi

$$
\frac{\Gamma(1-\alpha)}{n^{1-\alpha}}+\frac{\varphi(n, \alpha)}{n^{2-\alpha}},
$$

$\varphi(n, \alpha)$ étant une fonction bornée de $n$ et de $\alpha$ pour toutes les valeurs de $n$ et pour toutes les valeurs de $\alpha$ comprises dans l'intervalle $\left(-\frac{1}{2}, 0\right)$. Il suit de ces remarques qu'on peut poser

$$
\frac{d^{s} K_{n}(\alpha)}{d \alpha^{s}}=\frac{\Gamma(1-\alpha)}{n^{1-\alpha}}+\frac{\Psi^{s}(n, \alpha)}{n^{2-\alpha}},
$$

où la fonction $\psi(n, \alpha)$ est de la même nature que la fonction $\varphi(n, \alpha)$ tout à l'heure mentionnée. On a d'après la formule de Taylor

$$
\begin{aligned}
K_{n}(\alpha)= & K_{n}\left(-\frac{1}{2}\right)+\left(\alpha+\frac{1}{2}\right) K_{n}^{\prime}\left(-\frac{1}{2}\right)+\cdots \\
& +\left(\alpha+\frac{1}{2}\right)^{s-1} \frac{K_{n}^{(s-1)}\left(-\frac{1}{2}\right)}{(s-1) !}+\int_{-\frac{1}{2}}^{\alpha} \frac{(\alpha-x)^{s-1}}{(s-1) !} K_{n}^{(s)}(x) d x .
\end{aligned}
$$

Par ce qui précède on a uniformément en $\alpha$ $K_{n}\left(-\frac{1}{2}\right)+\left(\alpha+\frac{1}{2}\right) K_{n}^{\prime}\left(-\frac{1}{2}\right)+\cdots$

$$
+\left(\alpha+\frac{1}{2}\right)^{s-1} \frac{K_{n}^{(s-1)}\left(-\frac{1}{2}\right)}{(s-1) !}=O\left(1 / n^{3 / 2}\right) .
$$

D'autre part en substituant au lieu de $K_{n}^{(s)}(\alpha)$ son expression (6) on obtient

$$
\begin{aligned}
\int_{-\frac{1}{2}}^{\alpha} \frac{(\alpha-x)^{s-1}}{(s-1) !} \frac{d^{s} K_{n}(x)}{d x^{s}} d x= & \int_{-\frac{1}{2}}^{\alpha} \frac{(\alpha-x)^{s-1}}{(s-1) !} \frac{\Gamma(1-x)}{n^{1-x}} d x \\
& +\int_{-\frac{1}{2}}^{\alpha} \frac{(\alpha-x)^{s-1}}{(s-1) !} \frac{\psi(n, x)}{n^{2-x}} d x .
\end{aligned}
$$

La dernière intégrale est manifestement une quantité d'ordre $O\left(1 / n^{2}\right)$ indépendamment de $\alpha$. Quant à la première, l'appli- 
cation répétée de l'intégration par parties conduit à ce résultat

$$
\begin{aligned}
\int_{-\frac{1}{2}}^{\alpha} \frac{(\alpha-x)^{s-1}}{(s-1) !} & \frac{\Gamma(1-x)}{n^{1-x}} d x \\
& =\frac{\Gamma(1-\alpha)}{n^{1-\alpha}(\log n)^{s}}\left[1+\frac{\lambda(n, \alpha)}{\log n}\right]+O\left(1 / n^{3 / 2}\right),
\end{aligned}
$$

$\lambda(n, \alpha)$ désignant une fonction bornée de $n$ et de $\alpha$. Enfin nous arrivons à cette expression asymptotique de $K_{n}(\alpha)$ :

$$
K_{n}(\alpha)=\frac{\Gamma(1-\alpha)}{n^{1-\alpha}(\log n)^{s}}\left[1+\frac{\Lambda(n, \alpha)}{\log n}\right],
$$

la fonction $\Lambda(n, \alpha)$ étant de même nature que $\lambda(n, \alpha)$. En posant $\alpha=0$ nous voyons que

$$
\int_{0}^{h} \frac{(1-x)^{n} d x}{\left(\log \frac{1-x}{x}\right)^{s}} \sim \frac{1}{n(\log n)^{s}},
$$

ou d'une manière plus précise

$$
\int_{0}^{h} \frac{(1-x)^{n} d x}{\left(\log \frac{1-x}{x}\right)^{s}}=\frac{1}{n(\log n)^{s}}\left[1+\frac{\mu_{n}}{\log n}\right],
$$

$\mu_{n}$ désignant une fonction bornée de $n$.

4. Considérons maintenant l'intégrale

$$
\int_{-\infty}^{\infty} \frac{e^{h x} d x}{\pi^{2}+x^{2}}
$$

où $h$ est un nombre entier positif et $\omega$ un nombre positif quelconque. L'intégration par parties donne

(8) $\int_{-\infty}^{\infty} \frac{e^{h x} d x}{\pi^{2}+x^{2}}=\frac{e^{h \omega}}{h\left(\pi^{2}+\omega^{2}\right)}+\frac{2}{h} \int_{-\infty}^{\infty} \frac{e^{h x} x d x}{\left(\pi^{2}+x^{2}\right)^{2}}$.

En intégrant par parties de nouveau, nous avons

$$
\int_{-\infty}^{\infty} \frac{e^{h x} x d x}{\left(\pi^{2}+x^{2}\right)^{2}}=\frac{e^{h \omega} \omega}{h\left(\pi^{2}+\omega^{2}\right)^{2}}+\frac{1}{h} \int_{-\infty}^{\omega} \frac{e^{h x}\left(3 x^{2}-\pi^{2}\right)}{\left(\pi^{2}+x^{2}\right)^{3}} d x,
$$

mais comme 
val. abs. $\int_{-\infty}^{\infty} \frac{e^{h x}\left(3 x^{2}-\pi^{2}\right)}{\left(\pi^{2}+x^{2}\right)^{3}} d x<3 \int_{-\infty}^{\infty} \frac{e^{h x} d x}{\left(\pi^{2}+x^{2}\right)^{2}}$

$$
<\frac{3 e^{h \omega} \omega}{\left(\pi^{2}+\omega^{2}\right)^{2}}+\frac{3}{h \pi^{4}}<\frac{3 e^{h \omega}}{\omega^{3}}+\frac{3}{h \pi^{4}}<\frac{6 e^{h \omega}}{\omega^{3}},
$$

nous voyons que

$$
\text { val. abs. } \int_{-\infty}^{\omega} \frac{e^{h x} x d x}{\left(\pi^{2}+x^{2}\right)^{2}}<\frac{7 e^{h \omega}}{h \omega^{3}} .
$$

Cette inégalité combinée avec la formule (8) nous conduit à cette formule utile

$$
\int_{-\infty}^{\omega} \frac{e^{h x} d x}{\pi^{2}+x^{2}}=\frac{e^{h \omega}}{h\left(\pi^{2}+\omega^{2}\right)}+\frac{\mu e^{h \omega}}{h^{2} \omega^{3}}
$$

$\mu$ désignant une quantité dont la valeur absolue est toujours $<14$.

5. Ces préliminaires établis, désignons par $\xi_{0}$ une racine de l'équation

$$
\log \frac{1-\xi_{0}}{\xi_{0}}=1,
$$

qui est naturellement $>0$ et $<1 / 2$, et considérons l'intégrale

$$
\int_{0}^{\circ \xi_{0}} \xi^{k}(1-\xi)^{n-k} d \xi \int_{-\infty}^{\log \frac{1-\xi}{\xi}} \frac{e^{k x} d x}{\pi^{2}+x^{2}} .
$$

En utilisant la formule (9) nous aurons

$$
\begin{aligned}
& \int_{0}^{\xi_{0}} \xi^{k}(1-\xi)^{n-k} d \xi \int_{-\infty}^{\log \frac{1-\xi}{\xi}} \frac{e^{k x} d x}{\pi^{2}+x^{2}} \\
= & \frac{1}{k} \int_{0}^{\xi_{0}} \frac{(1-\xi)^{n} d \xi}{\pi^{2}+\left(\log \frac{1-\xi}{\xi}\right)^{2}}+\frac{M^{\prime}}{k^{2}} \int_{0}^{\xi_{0}} \frac{(1-\xi)^{n}}{\left(\log \frac{1-\xi}{\xi}\right)^{3}} d \xi .
\end{aligned}
$$

Mais

$$
\begin{aligned}
& \int_{0}^{\xi_{0}} \frac{(1-\xi)^{n} d \xi}{\pi^{2}+\left(\log \frac{1-\xi}{\xi}\right)^{2}} \\
= & \int_{0}^{\xi_{0}} \frac{(1-\xi)^{n} d \xi}{\left(\log \frac{1-\xi}{\xi}\right)^{2}}-\pi^{2} \int_{0}^{\rho \xi_{0}} \frac{(1-\xi)^{n} d \xi}{\left(\pi^{2}+\left(\log \frac{1-\xi}{\xi}\right)^{2}\right)\left(\log \frac{1-\xi}{\xi}\right)^{2}}
\end{aligned}
$$


et

$$
\int_{0}^{\rho \xi_{0}} \frac{(1-\xi)^{n} d \xi}{\left(\pi^{2}+\left(\log \frac{1-\xi}{\xi}\right)^{2}\right)\left(\log \frac{1-\xi}{\xi}\right)^{2}}<\int_{0}^{\partial \xi_{0}} \frac{(1-\xi)^{n} d \xi}{\left(\log \frac{1-\xi}{\xi}\right)^{4}}
$$

donc, en tenant compte de la formule asymptotique (7), nous avons d'abord

$$
\int_{0}^{\xi_{0}} \frac{(1-\xi)^{n} d \xi}{\pi^{2}+\left(\log \frac{1-\xi}{\xi}\right)^{2}} \sim \frac{1}{n(\log n)^{2}}
$$

et ensuite nous voyons que le rapport de l'intégrale

$$
\int_{0}^{\xi_{0}} \frac{(1-\xi)^{n} d \xi}{\left(\log \frac{1-\xi}{\xi}\right)^{3}} \dot{a} \int_{0}^{\xi_{0}} \frac{(1-\xi)^{n} d \xi}{\pi^{2}+\left(\log \frac{1-\xi}{\xi}\right)^{2}}
$$

est asymptotique à

$$
\frac{1}{\log n}
$$

ce qui nous conduit à la formule

$$
\begin{aligned}
\int_{0}^{\xi_{0}} \xi^{k}(1-\xi)^{n-k} d \xi \int_{-\infty}^{\log \frac{1-\xi}{\xi}} \frac{e^{k x} d x}{\pi^{2}+x^{2}} \\
=\frac{1}{k} \int_{0}^{\xi \xi_{0}} \frac{(1-\xi)^{n} d \xi}{\pi^{2}+\left(\log \frac{1-\xi}{\xi}\right)^{2}}\left[1+\frac{N}{k \log n}\right],
\end{aligned}
$$

où $N$ pour des valeurs suffisamment grandes de $n$ et quelque soit $k$ reste au dessous d'une limite assignable, p. e., 14. D'un autre côté, on trouve immédiatement

$$
\begin{aligned}
\int_{\xi_{0}}^{\frac{1}{2}} \xi k(1-\xi)^{n-k} d \xi \int_{-\infty}^{\log \frac{1-\xi}{\xi}} \frac{e^{k x} d x}{\pi^{2}+x^{2}} \\
<\frac{1}{k} \int_{\xi_{0}}^{\frac{1}{2}}(1-\xi)^{n} d \xi<\frac{\left(1-\xi_{0}\right)^{n+1}}{k(n+1)},
\end{aligned}
$$

et le rapport de cette quantité 


$$
\frac{\left(1-\xi_{0}\right)^{n+1}}{k(n+1)} \text { à } \frac{1}{k} \int_{0}^{\xi_{0}} \frac{(1-\xi)^{n} d \xi}{\pi^{2}+\left(\log \frac{1-\xi}{\xi}\right)^{2}}
$$

étant pour $n$ suffisamment grand plus petit que, p.e.e,

$$
2\left(1-\xi_{0}\right)^{n+1}(\log n)^{2}
$$

peut être mis sous la forme

$$
N^{\prime} /(k \log n) \quad \text { où } \quad N^{\prime}<2 n\left(1-\xi_{0}\right)^{n+1}(\log n)^{2}
$$

tend vers zéro $n$ grandissant indéfiniment. Ainsi nous avons enfin

ou bien

$$
\begin{aligned}
\int_{0}^{\frac{1}{2}} \xi^{k}(1-\xi)^{n-k} d \xi \int_{-\infty}^{\log \frac{1-\xi}{\xi}} \frac{e^{k x} d x}{\pi^{2}+x^{2}} & =\frac{1}{7} \int_{0}^{\xi_{0}} \frac{(1-\xi)^{n} d \xi}{\pi^{2}+\left(\log \frac{1-\xi}{\xi}\right)^{2}}\left[1+\frac{G}{k \log n}\right],
\end{aligned}
$$

$$
P_{1}=\frac{1}{k} \int_{0}^{\varsigma_{0}} \frac{(1-\xi)^{n} d \xi}{\pi^{2}+\left(\log \frac{1-\xi}{\xi}\right)^{2}}\left[1+\frac{G}{k \log n}\right],
$$

$G$ désignant une quantité qui reste au dessous de 15 pour $n$ suffisamment grand quelque soit $k$. De même

$$
P_{2}=\frac{1}{n-k} \int_{0}^{\xi_{0}} \frac{(1-\xi)^{n} d \xi}{\pi^{2}+\left(\log \frac{1-\xi}{\xi}\right)^{2}}\left[1+\frac{G^{\prime}}{(n-k) \log n}\right],
$$

où $G^{\prime}$ est aussi $<15$ pour $n$ assez grand quelque soit $k$. Il suffit maintenant d'introduire ces expressions dans la formule (4) pour avoir

$$
\begin{aligned}
J_{n}= & (-1)^{n-k} \boldsymbol{\Gamma}(k+1) \boldsymbol{\Gamma}(n+1-k)+\boldsymbol{\Gamma}(n+2) \\
\times & \left\{\int _ { 0 } ^ { \xi _ { 0 } } \frac { ( 1 - \xi ) ^ { n } d \xi } { \pi ^ { 2 } + ( \operatorname { l o g } \frac { 1 - \xi } { \xi } ) ^ { 2 } } \left[\frac{(-1)^{n-1}}{k}-\frac{1}{n-k}\right.\right. \\
& \left.\left.\quad+\frac{(-1)^{n-1} G}{k^{2} \log n}-\frac{G^{\prime}}{(n-k)^{2} \log n}\right]+\frac{\Theta}{k(n-k) 2^{n+1}}\right\}
\end{aligned}
$$


et aussi

$$
\begin{aligned}
& A_{k}= \frac{1}{n}+\frac{\Gamma(n+2)}{n \Gamma(k+1) \Gamma(n-k+1)} \\
& \times\left\{\int _ { 0 } ^ { \xi _ { 0 } } \frac { ( 1 - \xi ) ^ { n } d \xi } { \pi ^ { 2 } + ( \operatorname { l o g } \frac { 1 - \xi } { \xi } ) ^ { 2 } } \left[\frac{(-1)^{k-1}}{k}+\frac{(-1)^{n-k-1}}{n-k}\right.\right. \\
&\left.\left.\quad+\frac{(-1)^{k-1} G}{k^{2} \log n}+\frac{(-1)^{n-k-1} G^{\prime}}{(n-k)^{2} \log n}\right]+\frac{\Theta}{k(n-k) 2^{n+1}}\right\} .
\end{aligned}
$$

Le rapport de

$$
\frac{\Theta}{k(n-k) 2^{n+1}} \quad \grave{a} \frac{(-1)^{k-1}}{k}+\frac{(-1)^{n-k-1}}{n-k}
$$

est plus petit que

$$
\frac{1}{2^{n+1}\left|n-k+(-1)^{n} k\right|}<\frac{1}{2^{n+1}}
$$

car le nombre entier $n-k+(-1)^{n} k$ ne devient jamais égal à zéro. De même la valeur absolue du rapport de

$$
\frac{(-1)^{k-1} G}{k^{2} \log n}+\frac{(-1)^{n-k-1} G^{\prime}}{(n-k)^{2} \log n} \text { à } \frac{(-1)^{k-1}}{k}+\frac{(-1)^{n-k-1}}{n-k}
$$

pour $n$ suffisamment grand, est plus petite que

$$
\frac{15}{\log n} \cdot \frac{k^{2}+(n-k)^{2}}{k(n-k)\left|n-k+(-1)^{n} k\right|},
$$

c'est à dire pour $n$ pair

et pour $n$ impair

$$
\frac{15}{\log n} \cdot \frac{k^{2}+(n-k)^{2}}{k(n-k) n}
$$

$$
\frac{15}{\log n} \cdot \frac{k^{2}+(n-k)^{2}}{k(n-k)|n-2 k|} .
$$

Or quel que soit $k=1,2,3, \ldots, n-1$,

et

$$
\frac{k^{2}+(n-k)^{2}}{k(n-k) n}<1, n \text { pair }
$$

$$
\frac{k^{2}+(n-k)^{2}}{k(n-k)|n-2 k|}<3, n \text { impair }
$$


de sorte que le rapport précédent est toujours plus petit que

$$
\frac{45}{\log n}
$$

et tend vers zéro quand $n$ grandit indéfiniment. De tout ce qui précède il est maintenant facile de déduire cette expression asymptotique de $A_{k}$ :

$$
A_{k} \sim \frac{\Gamma(n+1)}{n(\log n)^{2} \boldsymbol{\Gamma}(k+1) \boldsymbol{\Gamma}(n-k+1)}\left[\frac{(-1)^{k-1}}{k}+\frac{(-1)^{n-k-1}}{n-k}\right],
$$

valable en ce sens que le rapport des deux membres de cette égalité tend uniformément vers 1 , quand $n$ croît au delà de toute limite, $k$ ayant une valeur quelconque $1,2,3, \cdots, n-1$. Quant aux coefficients $A_{0}$ et $A_{n}$, l'analyse toute semblable et même un peu plus simple donne

$$
A_{0}=A_{n} \sim \frac{1}{n \log n} .
$$

Ainsi nous avons

$$
A_{0} \sim \frac{1}{n \log n}, \quad A_{1} \sim \frac{1}{(\log n)^{2}}, \quad A_{2} \sim \frac{-n}{4(\log n)^{2}} \text { etc. }
$$

et pour $n=2 l$, p. e.,

$$
A_{l} \sim \frac{(-1)^{l-1} 4^{l}}{\sqrt{\pi} l^{5 / 2}(\log 2 l)^{2}} .
$$

On voit que les coefficients $A_{2}, A_{3}, \cdots, A_{n-2}$ tendent vers l'infini, ce qui fait voir que la formule de Cotes perd, toute valeur pratique tant que le nombre des ordonnées devient considérable.

Tononto, 25 Août, 1924. 